\title{
Quantitative phase-field modeling of faceted crystal dissolution processes
}

\author{
Nishant Prajapati, ${ }^{, \dagger, \uparrow}$ Michael Späth, ${ }^{\dagger}, \boldsymbol{\Phi}$ Linus Knecht, ${ }^{\dagger}$ Michael Selzer, ${ }^{\dagger, \ddagger}$ and \\ Britta Nestler ${ }^{\dagger, \ddagger}$ \\ $\dagger$ Institute for Applied Materials (IAM-CMS), Karlsruhe Institute of Technology (KIT), \\ Strasse am Forum 7, 76131 Karlsruhe, Germany \\ $\ddagger$ Institute for Digital Materials Science (IDM), Karlsruhe University of Applied Science, \\ Moltkestrasse 30, D-76133 Karlsruhe, Germany \\ ๑Authors contributed equally to this work \\ E-mail: nishant.prajapati@kit.edu
}

Abstract 


\section{Appendix}

This section lists the input set of vectors for the capillary and kinetic anisotropy functions for the simulations showcased in the present work. In all the simulations, the anisotropy strength parameter of $\delta=1000$ was set. Further, a list of all the symbols is given in the last part of this section. 


\section{A1. Anisotropy parameters for the hexagonal growth and dissolution forms}

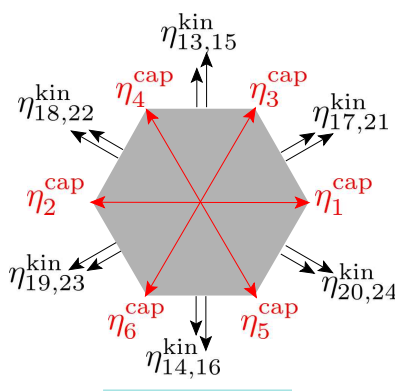

Dissolution form

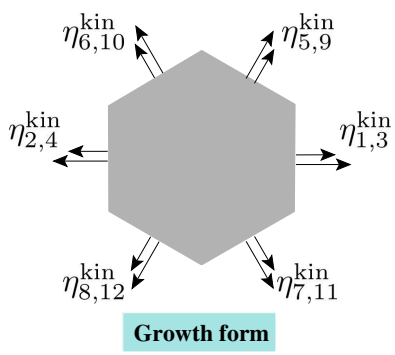

Figure A1: Hexagonal growth and dissolution forms illustrating the input vectors for the capillary and kinetic anisotropy corresponding to the vertices and facets of these forms.

\begin{tabular}{ccc}
\hline & $\mathrm{x}$ & $\mathrm{y}$ \\
\hline $\boldsymbol{\eta}_{1,2}^{\text {cap }}$ & \pm 1.155 & 0 \\
$\boldsymbol{\eta}_{3 . .6}^{\text {cap }}$ & \pm 0.577 & \pm 1 \\
\hline
\end{tabular}

Table A1: Set of vertex vectors for capillary anisotropy function corresponding to the 2-D hexagonal dissolution form, as also schematically shown (in red color) in figure A1.

\begin{tabular}{|c|c|c|c|c|c|}
\hline \multicolumn{3}{|c|}{ From growth form } & \multicolumn{3}{|c|}{ From dissolution form } \\
\hline & $\mathrm{x}$ & $\mathrm{y}$ & & $\mathrm{x}$ & $\mathrm{y}$ \\
\hline$\eta_{1,2}^{\mathrm{kin}}$ & \pm 1 & 0 & $\eta_{13,14}^{\mathrm{kin}}$ & 0 & \pm 1 \\
\hline$\eta_{3,4}^{\text {kin }}$ & $\pm 1^{*} \mathcal{A}_{1}$ & 0 & $\boldsymbol{\eta}_{15,16}^{\mathrm{kin}}$ & 0 & $\pm 1^{*} \mathcal{A}_{3}$ \\
\hline$\eta_{5 . .8}^{\text {kin }}$ & \pm 0.5 & \pm 0.866 & $\boldsymbol{\eta}_{17 . .20}^{\mathrm{kin}}$ & \pm 0.866 & \pm 0.5 \\
\hline$\eta_{9 . .12}^{\text {kin }}$ & $\pm 0.5^{*} \mathcal{A}_{2}$ & $\pm 0.866^{*} \mathcal{A}_{2}$ & $\eta_{21 . .24}^{\text {kin }}$ & $\pm 0.866^{*} \mathcal{A}_{4}$ & $\pm 0.5^{*} \mathcal{A}_{4}$ \\
\hline
\end{tabular}

Table A2: Facet normal vectors for kinetic anisotropy function for 2-D hexagonal growth and dissolution forms, as also schematically shown (in black color) in figure A1.

\section{A.2 Anisotropy parameters for Type B $\beta$-quartz}

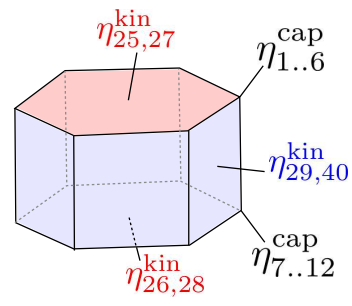

Dissolution form

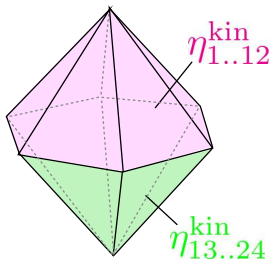

Growth form

Figure A2: Growth and dissolution forms of $\beta$-quartz illustrating the input vectors for the capillary and kinetic anisotropy corresponding to the vertices and facets of these forms.

\begin{tabular}{cccc}
\hline & x & y & z \\
\hline $\boldsymbol{\eta}_{1,2}^{\text {cap }}$ & \pm 1 & 0 & 1 \\
$\boldsymbol{\eta}_{3 . .6}^{\text {cap }}$ & \pm 0.5 & \pm 0.866 & 1 \\
$\boldsymbol{\eta}_{7,8}^{\text {cap }}$ & \pm 1 & 0 & -1 \\
$\boldsymbol{\eta}_{9 . .12}^{\text {cap }}$ & \pm 0.5 & \pm 0.866 & -1 \\
\hline
\end{tabular}

Table A3: Set of vertex vectors for capillary anisotropy function corresponding to the $\beta$ quartz dissolution form, as also schematically shown (in black color) in figure A2. 
Table A4: Facet normal vectors for kinetic anisotropy function for $\beta$-quartz growth and dissolution forms, as also schematically shown in figure A2.

\begin{tabular}{|c|c|c|c|c|c|c|c|}
\hline \multicolumn{4}{|c|}{ From growth form } & \multicolumn{4}{|c|}{ From dissolution form } \\
\hline & $\mathrm{x}$ & $\mathrm{y}$ & $\mathrm{Z}$ & & $\mathrm{x}$ & $\mathrm{y}$ & $\mathrm{z}$ \\
\hline $\boldsymbol{\eta}_{1 . .2}^{\mathrm{kin}}$ & \pm 0.7857 & 0 & 0.6186 & $\boldsymbol{\eta}_{25,26}^{\mathrm{kin}}$ & 0 & 0 & \pm 1 \\
\hline $\boldsymbol{\eta}_{3 . .6}^{\mathrm{kin}}$ & \pm 0.3929 & \pm 0.6805 & 0.6186 & $\boldsymbol{\eta}_{27,28}^{\text {kin }}$ & 0 & 0 & $\pm 1^{*} \mathcal{A}_{c}$ \\
\hline $\boldsymbol{\eta}_{7.8}^{\text {kin }}$ & $\pm 0.7857^{*} \mathcal{A}_{d}$ & 0 & $0.6186^{*} \mathcal{A}_{d}$ & $\boldsymbol{\eta}_{2930}^{\mathrm{kin}}$ & \pm 1 & 0 & 0 \\
\hline $\boldsymbol{\eta}_{9 . .12}^{\mathrm{kin}}$ & $\pm 0.3929 * \mathcal{A}_{d}$ & $\pm 0.6805^{*} \mathcal{A}_{d}$ & $0.6186^{*} \mathcal{A}_{d}$ & $\boldsymbol{\eta}_{31,32}^{\mathrm{kin}}$ & $\pm 1^{*} \mathcal{A}_{m}$ & 0 & 0 \\
\hline $\boldsymbol{\eta}_{13 . .14}^{\mathrm{kin}}$ & \pm 0.7857 & 0 & -0.6186 & $\boldsymbol{\eta}_{33.36}^{\text {kin }}$ & \pm 0.866 & \pm 0.5 & 0 \\
\hline $\boldsymbol{\eta}_{15.18}^{\text {kin }}$ & \pm 0.3929 & \pm 0.6805 & -0.6186 & $\boldsymbol{\eta}_{37.40}^{\text {kin }}$ & $\pm 0.866^{*} \mathcal{A}_{m}$ & $\pm 0.5^{*} \mathcal{A}_{m}$ & 0 \\
\hline $\boldsymbol{\eta}_{19 . .20}^{\text {kin }}$ & $\pm 0.7857^{*} \mathcal{A}_{d}$ & 0 & $-0.6186^{*} \mathcal{A}_{d}$ & & & & \\
\hline $\boldsymbol{\eta}_{21 . .24}^{\text {kin }}$ & $\pm 0.3929^{*} \mathcal{A}_{d}$ & $0.6805^{*} \mathcal{A}_{d}$ & $-0.6186^{*} \mathcal{A}_{d}$ & & & & \\
\hline
\end{tabular}

\section{A.3 Anisotropy parameters for $\beta$-succinic acid}
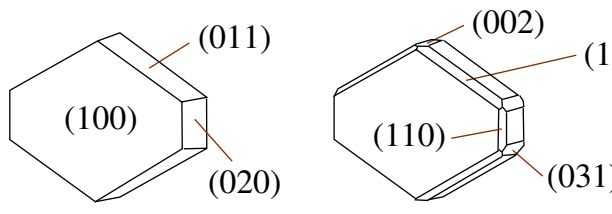

Growth form
Intermediate shape

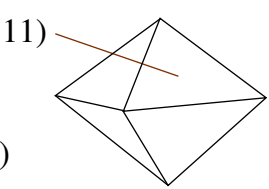

Dissolution form

\begin{tabular}{cccc}
\hline & x & y & z \\
\hline $\boldsymbol{\eta}_{1,2}^{\text {cap }}$ & \pm 1 & 0 & 0 \\
$\boldsymbol{\eta}_{3,4}^{\text {cap }}$ & 0 & \pm 1 & 0 \\
$\boldsymbol{\eta}_{5,6}^{\text {cap }}$ & 0 & 0 & \pm 1 \\
\hline
\end{tabular}

Figure A3: Growth and dissolution forms of $\beta$-succinic acid along with the intermediate shape with additional facets appearing during dissolution.

Table A5: Set of vertex vectors for capillary anisotropy function corresponding to the $\beta$ succinic acid dissolution form.

Table A6: Facet normal vectors for kinetic anisotropy function for the growth and dissolution forms along with intermediate shape for $\beta$-succinic acid, as shown in figure A3. The subscript of each anisotropy strength parameter appearing in the components of a vector corresponds to the facet it belongs to.

\begin{tabular}{|c|c|c|c|c|c|c|c|c|c|c|}
\hline \multicolumn{4}{|c|}{ From growth form } & \multicolumn{3}{|c|}{ From dissolution form } & \multicolumn{4}{|c|}{ From intermediate shape } \\
\hline & $\mathrm{x}$ & $\mathrm{y}$ & $\mathrm{z}$ & $\mathrm{x}$ & $\mathrm{y}$ & $\mathrm{z}$ & & $\mathrm{x}$ & $\mathrm{y}$ & $\mathrm{z}$ \\
\hline $\boldsymbol{\eta}_{1,2}^{\mathrm{kin}}$ & \pm 1 & 0 & 0 & \pm 0.5774 & \pm 0.5774 & \pm 0.5774 & $\boldsymbol{\eta}_{33.36}^{\mathrm{kin}}$ & \pm 0.9487 & \pm 0.3162 & 0 \\
\hline $\boldsymbol{\eta}_{3,4}^{\mathrm{kin}}$ & $\pm 1 * \mathcal{A}_{020}$ & 0 & 0 & $\boldsymbol{\eta}_{25.32}^{\mathrm{kin}} \pm 0.5774 * \mathcal{A}_{111}$ & $\pm 0.5774 * \mathcal{A}_{111}$ & $\pm 0.5774 * \mathcal{A}_{111}$ & $\boldsymbol{\eta}_{37 . .40}^{\mathrm{kin}}$ & $\pm 0.9487 * \mathcal{A}_{031}$ & $\pm 0.3162 * \mathcal{A}_{031}$ & 0 \\
\hline $\boldsymbol{\eta}_{5,6}^{\mathrm{kin}}$ & 0 & 0 & \pm 1 & & & & $\boldsymbol{\eta}_{41 . .44}^{\mathrm{kin}}$ & \pm 0.7071 & 0 & \pm 0.7071 \\
\hline $\boldsymbol{\eta}_{7,8}^{\mathrm{kin}}$ & 0 & 0 & $\pm 1 * \mathcal{A}_{100}$ & & & & $\boldsymbol{\eta}_{45 . .48}^{\mathrm{kin}}$ & $\pm 0.7071 * \mathcal{A}_{101}$ & 0 & $\pm 0.7071 * \mathcal{A}_{101}$ \\
\hline$\eta_{9.12}^{\mathrm{kin}}$ & \pm 0.7071 & \pm 0.7071 & 0 & & & & $\boldsymbol{\eta}_{49.52}^{\mathrm{kin}}$ & 0 & \pm 0.7071 & \pm 0.7071 \\
\hline \multirow[t]{3}{*}{$\boldsymbol{\eta}_{13 \ldots 16}^{\text {kin }}$} & $\pm 0.7071 * \mathcal{A}_{011}$ & $\pm 0.7071 * \mathcal{A}_{011}$ & 0 & & & & $\boldsymbol{\eta}_{53.56}^{\text {kin }}$ & 0 & $\pm 0.7071 * \mathcal{A}_{011}$ & $\pm 0.7071 * \mathcal{A}_{011}$ \\
\hline & & & & & & & $\boldsymbol{\eta}_{57,58}^{\mathrm{kin}}$ & 0 & \pm 1 & 0 \\
\hline & & & & & & & $\boldsymbol{\eta}_{59,60}^{\text {kin }}$ & 0 & $\pm 1 * \mathcal{A}_{002}$ & 0 \\
\hline
\end{tabular}




\section{A.4. Anisotropy parameters for $\alpha$-quartz}

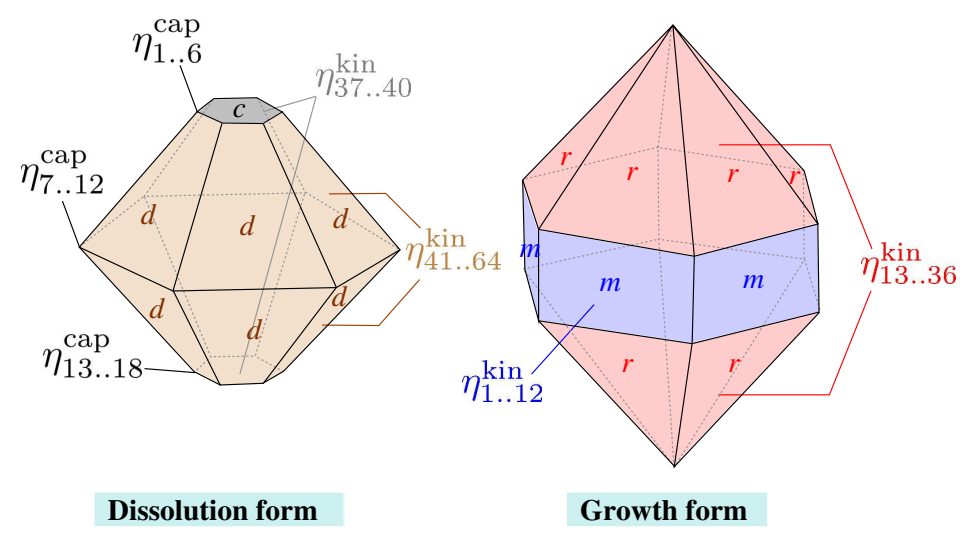

Figure A4: Growth and dissolution forms of $\alpha$-quartz illustrating the subscript-numbering of the input vectors for the capillary and kinetic anisotropy corresponding to the vertices and facets of these forms.

\begin{tabular}{cccc}
\hline & $\mathrm{x}$ & $\mathrm{y}$ & $\mathrm{z}$ \\
\hline $\boldsymbol{\eta}_{1,2}^{\text {cap }}$ & \pm 0.5105 & 0 & 1 \\
$\boldsymbol{\eta}_{3 . .6}^{\text {cap }}$ & \pm 0.2552 & \pm 0.4421 & 1 \\
$\boldsymbol{\eta}_{7,8}^{\text {cap }}$ & \pm 1.5612 & 0 & 0 \\
$\boldsymbol{\eta}_{9 . .12}^{\text {cap }}$ & \pm 0.7806 & \pm 1.352 & 0 \\
$\boldsymbol{\eta}_{13,14}^{\text {cap }}$ & \pm 0.5105 & 0 & -1 \\
$\boldsymbol{\eta}_{15 . .18}^{\text {cap }}$ & \pm 0.2552 & \pm 0.4421 & -1 \\
\hline
\end{tabular}

Table A7: Set of vertex vectors for capillary anisotropy function corresponding to the $\alpha$ quartz dissolution form.

Table A8: Facet normal vectors for kinetic anisotropy function for the growth and dissolution forms along with additional vectors (to avoid non-physical curvatures) for $\alpha$-quartz, as also schematically shown in figure A4.

\begin{tabular}{|c|c|c|c|c|c|c|c|c|c|c|c|}
\hline \multicolumn{4}{|c|}{ From growth form } & \multicolumn{4}{|c|}{ From dissolution form } & \multicolumn{4}{|c|}{ Additional vectors } \\
\hline & $\mathrm{x}$ & $\mathrm{y}$ & $\mathrm{z}$ & & $\mathrm{x}$ & $\mathrm{y}$ & $\mathrm{Z}$ & & $\mathrm{x}$ & $\mathrm{y}$ & $\mathrm{Z}$ \\
\hline $\boldsymbol{\eta}_{1,2}^{\mathrm{kin}}$ & \pm 1 & 0 & 0 & $\boldsymbol{\eta}_{37,38}^{\mathrm{kin}}$ & 0 & 0 & \pm 1 & $\boldsymbol{\eta}_{65 . .68}^{\mathrm{kin}}$ & 0 & \pm 1 & \pm 0.22 \\
\hline $\boldsymbol{\eta}_{3,4}^{\mathrm{kin}}$ & $\pm 1^{*} \mathcal{A}_{m}$ & 0 & 0 & $\boldsymbol{\eta}_{39,40}^{\mathrm{kin}}$ & 0 & 0 & $\pm 1^{*} \mathcal{A}_{c}$ & $\boldsymbol{\eta}_{69.72}^{\mathrm{kin}}$ & 0 & $\pm 1^{*} \mathcal{A}_{a}$ & $\pm 0.22^{*} \mathcal{A}_{a}$ \\
\hline $\boldsymbol{\eta}_{5}^{\mathrm{kin}}$ & \pm 0.5 & \pm 0.866 & 0 & $\eta_{41}^{\text {kin }}$ & 0 & \pm 0.74 & \pm 0.6727 & $\boldsymbol{\eta}_{73}^{\text {kin }}$ & \pm 0.866 & \pm 0.5 & \pm 0.22 \\
\hline $\boldsymbol{\eta}_{9 . .12}^{\mathrm{kin}^{8}}$ & $\pm 0.5^{*} \mathcal{A}_{m}$ & $\pm 0.866^{*} \mathcal{A}_{m}$ & 0 & $\boldsymbol{\eta}_{45 . .48}^{\mathrm{kin}}$ & 0 & $\pm 0.74^{*} \mathcal{A}_{d}$ & $\pm 0.6727^{*} \mathcal{A}_{d}$ & $\boldsymbol{\eta}_{81 . .88}^{\mathrm{kin}}$ & $\pm 0.866^{*} \mathcal{A}_{a}$ & $\pm 0.5^{*} \mathcal{A}_{a}$ & $\pm 0.22^{*} \mathcal{A}_{a}$ \\
\hline $\boldsymbol{\eta}_{13}^{\mathrm{kin}}$ & \pm 0.7857 & 0 & \pm 0.6186 & $\boldsymbol{\eta}_{49}^{\mathrm{kin}}$ & \pm 0.6408 & \pm 0.37 & \pm 0.6727 & & & & \\
\hline $\begin{array}{l}\boldsymbol{\eta}_{17 . .20}^{\mathrm{kin}} \\
\boldsymbol{\eta}_{21 \ldots 28}^{\mathrm{kin}} \\
\boldsymbol{\eta}_{29 . .36}^{\mathrm{kin}}\end{array}$ & $\begin{array}{l} \pm 0.7857^{*} \mathcal{A}_{r} \\
\quad \pm 0.3929 \\
\pm 0.3929 * \mathcal{A}_{r}\end{array}$ & $\begin{array}{c}0 \\
\pm 0.6805 \\
\pm 0.6805^{*} \mathcal{A}_{r}\end{array}$ & $\begin{array}{l} \pm 0.6186^{*} \mathcal{A}_{r} \\
\quad \pm 0.6186 \\
\pm 0.6186^{*} \mathcal{A}_{r}\end{array}$ & $\eta_{57 . .64}^{\text {kin }}$ & $\pm 0.6408^{*} \mathcal{A}_{d}$ & $\pm 0.37^{*} \mathcal{A}_{d}$ & $\pm 0.6727^{*} \mathcal{A}_{d}$ & & & & \\
\hline
\end{tabular}

\section{B List of Symbols}

Complete list of symbols corresponding to all the physical and model parameters used in this work is given in table A9. 
Table A9: List of Symbols. $(\cdot)$ denotes the place-holder for one phase, $(\cdot \cdot)$ is the place-holder for two phases and $(*)$ represents the place-holder for a facet.

\begin{tabular}{|c|c|c|c|}
\hline Model parameters & Symbol & Subscript of phase $(\cdot)$ & Symbol \\
\hline Phase-field variable of phase $(\cdot)$ & $\phi_{(\cdot)}$ & solid & $s$ \\
\hline Phase-field duplet & $\phi$ & liquid & $l$ \\
\hline Physical domain & $\Omega$ & quartz & q \\
\hline Grid cell size & $\Delta x$ & water & $\mathrm{w}$ \\
\hline Time step width & $\Delta t$ & \multicolumn{2}{|l|}{ Subscript of two phases $(. \cdot)$} \\
\hline Bulk region of phase $(\cdot)$ & $\mathcal{B}_{(\cdot)}$ & solid-liquid & $s l$ \\
\hline Diffuse interface region between phases $(. \cdot)$ & $\mathcal{I}_{(. \cdot)}$ & quartz-water & qw \\
\hline Helmholtz free energy & $\mathcal{F}$ & \multicolumn{2}{|l|}{$\alpha$-Quartz specific model parameters } \\
\hline Bulk free energy contribution & $\mathcal{F}_{\text {bulk }}$ & Temperature & $T$ \\
\hline Interface free energy contribution & $\mathcal{F}_{\text {interface }}$ & Pressure & $p$ \\
\hline Bulk free energy density & $\mathfrak{f}_{\text {bulk }}$ & Difference of molar volume & $\Delta V$ \\
\hline Interface free energy density & $f_{\text {interface }}$ & Change of Gibbs free energy & $\Delta G$ \\
\hline Free energy density of phase $(\cdot)$ & $\mathfrak{f}_{(\cdot)}$ & Gas constant & $R$ \\
\hline Potential energy density & $\omega / \varepsilon$ & Saturation index & $\mathcal{S}$ \\
\hline Gradient energy density & $\varepsilon a$ & Equilibrium concentration of $\mathrm{H}_{4} \mathrm{SiO}_{4}$ & $\mathfrak{c}_{\mathrm{H}_{4} \mathrm{SiO}_{4}}^{\mathrm{eq}}$ \\
\hline Length scale parameter & $\varepsilon$ & Actual concentration of $\mathrm{H}_{4} \mathrm{SiO}_{4}$ & $\mathfrak{c}_{\mathrm{H}_{4} \mathrm{SiO}_{4}}$ \\
\hline Surface energy of (..) interface & $\gamma_{(. .)}$ & Dissolution rate constant for quartz & $k_{+}$ \\
\hline Capillary anisotropy function & $a_{(.)}^{\text {cap }}$ & Molar volume of quartz & $V_{\mathrm{m}}^{\mathrm{qtz}}$ \\
\hline Phase-field gradient vector & $\mathbf{q}_{(\cdot .)}$ & Molar volume of water & $V_{\mathrm{m}}^{\mathrm{H}_{2} \mathrm{O}}$ \\
\hline Unit phase-field gradient vector & $\mathfrak{n}_{(. .)}$ & Velocity of qw interface & $v_{\mathrm{qw}}$ \\
\hline Kinetic mobility of $(. \cdot)$ interface & $\mu_{(. .)}$ & Velocity of $m, r, d, c$-facet & $v_{(*)}$ \\
\hline Kinetic coefficient of $(\cdot$.$) interface$ & $\mu_{(. .)}^{0}$ & & \\
\hline Kinetic anisotropy function of $(. \cdot)$ interface & $a_{(\cdot .)}^{\text {kin }}$ & & \\
\hline Capillary anisotropy vector set & $\mathcal{N}^{\text {cap }}$ & & \\
\hline$k^{\text {th }}$ Capillary anisotropy vector & $\eta_{k}^{\text {cap }}$ & & \\
\hline Kinetic anisotropy vector set & $\mathcal{N}^{\text {kin }}$ & & \\
\hline$k^{\text {th }}$ Kinetic anisotropy vector & $\boldsymbol{\eta}_{k}^{\text {kin }}$ & & \\
\hline Full set of anisotropy strength parameters & $\hat{\mathcal{A}}$ & & \\
\hline Reduced set of anisotropy strength parameters & $\mathcal{A}$ & & \\
\hline Facet specific anisotropy strength parameter & $\mathcal{A}_{(*)}$ & & \\
\hline Driving force for dissolution & $\Delta f_{(.)}^{\mathrm{Diss}}$ & & \\
\hline
\end{tabular}

